\title{
Plants used as painkiller in traditional treatment in Turkey - II Headache
}

\author{
Meryem Şeyda Erbay*, Sezin Anıl, Gülay Melikoğlu \\ Istanbul University, Faculty of Pharmacy, Department of Pharmacognosy, Faculty of Pharmacy, Istanbul University, Beyazit, Istanbul, Turkey.
}

\begin{abstract}
Turkey has many plants used by public for the treatment of various diseases. The usage of these plants has been transferred from generation to generation. Ethnobotanical studies carried out by traditional methods of treatment are recorded and this information is aimed to contribute to the development of the drug. In this study, it has been reached to 88 taxa that is used in traditional treatment against headache in Turkey and aimed to give information about scientific and local names, families, used parts and usage of these taxa in headache. in headache.
\end{abstract}

KEYWORDS: Headache, medicinal plants, traditional treatment, Turkey.

\section{INTRODUCTION}

Pain is an important health problem that affects millions of people every year and develops due to various causes and has a negative impact on quality of life. International health care organizations have defined pain as a sign of disease [1]. It is also accepted that pain is a disease in itself [2].

Turkey has a great diversity in terms of climate and geographical conditions. This diversity brings the advantage of having a rich plant cover. Approximately eleven thousand plant species are growing in our country, and three thousand of them are endemic [3-5]. From the early ages, the plants are used by people as food or to prevent health problems. Medicinal plants, which have been traditionally used by the public at the time, are recorded with ethnobotanical investigations. Such studies are a very important source of information for drug research. Folk medicine has an important place both in the world and in our country in health field. Traditional treatment is frequently used in the treatment of diseases, especially in less developed regions. In this study, plants which have traditionally been used as painkillers in Turkey have been identified by screening ethnobotanical studies. The findings are classified according to pain types and will be published as an article series. This study, which is second article of series, contains the plants used against headache.

Headache is the most common type of sickness. It may be caused by cold and fatigue or can be a symptom of a serious illness. Some disorders such as ocular disorders, high blood

Received: 31.01.2017/ Accepted: 08.05.2017

Corresponding Author: Meryem Şeyda Erbay

E-mail: mseydaerbay@gmail.com

ORCID No: https://orcid.org/0000-0001-9889-9250 pressure, presence of urine in the head and neck region, meningitis, sinusitis, otitis, head trauma and tension lead to headaches. For the treatment of continuous headache, sudden onset of severe headache, or headache that causes visual and hearing complaints, drowsiness in crows and stools, daytime headache and aggravated headaches, time should not be wasted by self-treatment and should be referred to a doctor [6]. In simple headaches, various plants can be used to relieve pain.

\section{MATERIAL AND METHODS}

This study is prepared by searching thesis at the Council of Higher Education theses Center and ethnobotanical studies conducted in various parts of the Turkey with selecting plants used for headache.

\section{RESULTS AND DISCUSSION}

This study reached 88 taxa traditionally used against headache in Turkey. The information of the plant taxa (scientific and local names, families, used parts and usage in headache] in this research is given in Table 1.

How to cite this article: Erbay MŞ, Anıl S, Melikoğlu G. Plants used as painkiller in traditional treatment in Turkey II - Headache. Marmara Pharm J. 2018; 22 (1) : 29-36. 
Table 1. The plants used in traditional treatment against headache in Turkey.

\begin{tabular}{|c|c|c|c|c|c|}
\hline Botanical name & Family & Local name & Plant part used & $\begin{array}{l}\text { Preparation, } \\
\text { administration and } \\
\text { use }\end{array}$ & Ref. \\
\hline $\begin{array}{l}\text { Achillea biebersteinii } \\
\text { Afan. }\end{array}$ & Asteraceae & $\begin{array}{l}\text { Arı çiçeği, Erkurtaran, } \\
\text { Gırtkesan, Çiçege maran, } \\
\text { Sarı çiçek, Ayvadana, } \\
\text { Sancı çiçeği }\end{array}$ & Aerial part & Inf., Int. & [7] \\
\hline $\begin{array}{l}\text { A. millefolium L. subsp. } \\
\text { pannonica (Scheele) } \\
\text { Hayek }\end{array}$ & Asteraceae & $\begin{array}{l}\text { Ayvadana, Civanperçemi, } \\
\text { Kurpotu, Ronağvaç, } \\
\text { Sporiş, Krannavaz }\end{array}$ & Leaf & Dec., Int. & [8] \\
\hline $\begin{array}{l}\text { A. nobilis L. subsp. } \\
\text { neilreichii (Kerner) } \\
\text { Formanek }\end{array}$ & Asteraceae & $\begin{array}{l}\text { Civanperçemi, Ayvadana, } \\
\text { Aslan bıyığı, Kurt otu }\end{array}$ & Aerial part & Dec., Int. & [9] \\
\hline Acorus calamus L. & Araceae & Eğir, Hazanbel, Azak eğiri & Root & Inf. & {$[10]$} \\
\hline $\begin{array}{l}\text { Alcea hohenackeri } \\
\text { (Boiss.\&Huet.) Boiss. }\end{array}$ & Malvaceae & Hero & Root & Dec., Int. & {$[11]$} \\
\hline Allium cepa L. & Amaryllidaceae & $\begin{array}{l}\text { Soğan, Pivaz, Kıska, } \\
\text { Arpacık soğanı, } \\
\text { Karacasoğan }\end{array}$ & Stem, Root and Leaf & Eaten & {$[12]$} \\
\hline A. sativum $\mathrm{L}$. & Amaryllidaceae & Sarımsak & Bulb & $\begin{array}{l}\text { Eaten } \\
\text { Crushed, Ext. }\end{array}$ & $\begin{array}{l}{[13 ; 12]} \\
{[9]}\end{array}$ \\
\hline Anethum graveolens L. & Apiaceae & $\begin{array}{l}\text { Tere otu, Dereotu, } \\
\text { Arapsaçı, Çarşır, Kırca, } \\
\text { Tere }\end{array}$ & Seed & Eaten & [9] \\
\hline $\begin{array}{l}\text { Arum conophalloides } \\
\text { Kotschy ex Schott }\end{array}$ & Araceae & Yılan bıçağı & Leaf & Heated, Ext. & {$[14]$} \\
\hline A. elongatum Steven & Araceae & $\begin{array}{l}\text { El kabartan, Gavur otu, } \\
\text { Kabarcık, Kabarağı, } \\
\text { Yllancık, Yılan dili }\end{array}$ & Leaf & Ext. & {$[15]$} \\
\hline Artemisia absinthium L. & Asteraceae & $\begin{array}{l}\text { Granguruh, Tahliş, Pelin } \\
\text { otu, Acı yavşan }\end{array}$ & Aerial part & Dec., Int. & {$[11]$} \\
\hline A. spicigera C.Koch & Asteraceae & Glyabend & Aerial part & Dec., Int. & {$[16]$} \\
\hline $\begin{array}{l}\text { Ballota nigra L. subsp. } \\
\text { anatolica P.H. Davis }\end{array}$ & Lamiaceae & Arı otu & Leaf & Dec., Int. & {$[17]$} \\
\hline Bellis perennis L. & Asteraceae & $\begin{array}{l}\text { Yoğurt çiçeği, Papatya, } \\
\text { Beyaz papatya }\end{array}$ & $\begin{array}{l}\text { Aerial part } \\
\text { Whole plant } \\
\text { Flower }\end{array}$ & $\begin{array}{l}\text { Dec., Int. } \\
\text { +Lawsonia inermis, Ext. } \\
\text { Not stated }\end{array}$ & $\begin{array}{l}{[18]} \\
{[19]} \\
{[20]}\end{array}$ \\
\hline Buxus sempervirens L. & Buxaceae & Şimşir & Leaf & Dec., Int. & [21] \\
\hline $\begin{array}{l}\text { Cerasus avium (L.) } \\
\text { Moench }\end{array}$ & Rosaceae & Kiraz & Fruit & Fresh or dried eaten & [11] \\
\hline $\begin{array}{l}\text { C. brachypetala } \\
\text { Boiss. var. } \\
\text { bornmuelleri(Schneider) } \\
\text { Browicz }\end{array}$ & Rosaceae & Helhelok & Fruit & Fresh or dried eaten & [11] \\
\hline Cistus creticus L. & Cistaceae & $\begin{array}{l}\text { Pambulcuk, Pamukla otu, } \\
\text { Çoban çırası, Karahan, } \\
\text { Garahan }\end{array}$ & Aerial part & Incense, Ext. & [9] \\
\hline $\begin{array}{l}\text { Citrus limonum (L.) } \\
\text { Burnm. fil. }\end{array}$ & Rutaceae & Limon & Pericarp & Ext. & {$[22]$} \\
\hline C. sinensis (L.) Osbeck & Rutaceae & Portakal & Pericarp & Ext. & {$[22]$} \\
\hline Coffeae arabica $\mathrm{L}$. & Rubiaceae & Kahve & Seed & Inf., Int. & {$[23]$} \\
\hline
\end{tabular}




\begin{tabular}{|c|c|c|c|c|c|}
\hline Cornus mas L. & Cornaceae & Kızılcık, Püren & Fruit & Paste, Int. & {$[24]$} \\
\hline $\begin{array}{l}\text { Crataegus aronia (L.) } \\
\text { Bosc.ex DC. var. aronia }\end{array}$ & Rosaceae & Sarı alıç, Ahlat, Alıç & Fruit & Crushed, +Sugar, Int. & {$[22]$} \\
\hline Cucumis sativus $\mathrm{L}$. & Cucurbitaceae & $\begin{array}{l}\text { Salatalık, Siğilli salatalık, } \\
\text { Acur }\end{array}$ & Fruit & $\begin{array}{l}\text { Eaten on an empty } \\
\text { stomach }\end{array}$ & [9] \\
\hline Cydonia oblonga Miller & Rosaceae & Ayva, Verekılfercel & Leaf & Dec., Int. & {$[8 ; 25 ; 26]$} \\
\hline $\begin{array}{l}\text { Cynodon dactylon (L.) } \\
\text { Pers. var.villosus Regel }\end{array}$ & Poaceae & Ayrık, Ayrık otu & Rhizome & Chewed, Int. & {$[13]$} \\
\hline $\begin{array}{l}\text { Dracunculus vulgaris } \\
\text { Schott }\end{array}$ & Araceae & $\begin{array}{l}\text { Yılan pancarı, Yılan ebesi, } \\
\text { Yılan burçağı, Yılanbaşı, } \\
\text { Yılan darısı }\end{array}$ & Leaf & Ext. & {$[27]$} \\
\hline $\begin{array}{l}\text { Ecballium elaterium (L.) } \\
\text { A. Rich. }\end{array}$ & Cucurbitaceae & $\begin{array}{l}\text { Acı kavun, Şeytan } \\
\text { kavunu, Cırtlak, Eşek } \\
\text { hıyarı, Ebuceyil kavunu, } \\
\text { Gargadüneği, Acı bostan, } \\
\text { Acı kelek, Şeytan keleği }\end{array}$ & $\begin{array}{l}\text { Fruit juice } \\
\text { Root }\end{array}$ & $\begin{array}{l}\text { Dropped into the } \\
\text { nostrils } \\
\text { Crushed, Ext. }\end{array}$ & $\begin{array}{l}{[9 ; 28]} \\
{[29]}\end{array}$ \\
\hline Ephedra major Host & Ephedraceae & Deniz üzümü & Fruit & Inf., Int. & {$[30]$} \\
\hline $\begin{array}{l}\text { Eremurus spectabilis } \\
\text { Bieb. }\end{array}$ & Liliaceae & Helige, Çiriş & Root & Crushed, Ext. & {$[31 ; 32]$} \\
\hline $\begin{array}{l}\text { Ficus carica L. subsp. } \\
\text { carica }\end{array}$ & Moraceae & İncir & Leaf & Ext. & {$[15]$} \\
\hline $\begin{array}{l}\text { Fritillaria acmopetala } \\
\text { Boiss. subsp. wendelboi } \\
\text { Pax }\end{array}$ & Liliaceae & Ağlayan gelin & Bulb & Cooked, Mush, Ext. & [33] \\
\hline $\begin{array}{l}\text { Glaucium leiocarpum } \\
\text { Boiss. }\end{array}$ & Papaveraceae & Gelincik & Leaf & Ext., Cigaret & {$[30 ; 34]$} \\
\hline Hordeum murinum L. & Poaceae & Kılçık otu, Arpa & Leaf & Eaten & [35] \\
\hline Hypericum scabrum L. & Hypericaceae & Kantaron & Aerial part & Inf., Int. & {$[12]$} \\
\hline Inula heterolepis Boiss. & Asteraceae & Caye röe, Çayotu & Aerial part & Inf., Int. & {$[30 ; 36]$} \\
\hline Juglans regia L. & Juglandaceae & Ceviz & $\begin{array}{l}\text { Leaf } \\
\text { Pericarp }\end{array}$ & $\begin{array}{l}\text { Mush, Ext. } \\
\text { +Lawsonia inermis, Ext. } \\
\text { Dec., Int. }\end{array}$ & $\begin{array}{l}{[37]} \\
{[15]} \\
{[38]}\end{array}$ \\
\hline Juniperus oxycedrus L. & Cupressaceae & Ardıç & Fruit & $\begin{array}{l}\text { Eaten, In the morning } \\
\text { on an empty stomach }\end{array}$ & {$[15]$} \\
\hline $\begin{array}{l}\text { Lagenaria siceraria } \\
\text { (Mol.) Standl. var. } \\
\text { clavata }\end{array}$ & Cucurbitaceae & Su kabağ 1 & Leaf & Ext. & {$[22]$} \\
\hline $\begin{array}{l}\text { Laurocerasus officinalis } \\
\text { Roem. }\end{array}$ & Rosaceae & Karayemiş & Leaf & Eaten & [39] \\
\hline $\begin{array}{l}\text { Lavandula officinalis } \\
\text { Chaix }\end{array}$ & Lamiaceae & Lavanta & Aerial part & Inf. & {$[10]$} \\
\hline L. stoechas L. & Lamiaceae & Karabaş otu, Kocabaş otu & Aerial part & Dec., Int. & {$[14 ; 21 ; 40]$} \\
\hline $\begin{array}{l}\text { L. stoechas L. subsp. } \\
\text { stoechas }\end{array}$ & Lamiaceae & $\begin{array}{l}\text { Karahan, Karabaş, } \\
\text { Karabaş otu, Dede burnu, } \\
\text { Karaburun, Morbaş }\end{array}$ & Flowering branch & Inf., Int. & {$[41]$} \\
\hline Lycopodium clavatum $\mathrm{L}$. & Lycopodiaceae & Aslan pençesi & Leaf & Inf./Dec. & {$[21]$} \\
\hline $\begin{array}{l}\text { Malus slyvestris Mill. } \\
\text { subsp. orientalis } \\
\text { (uglitzk.) Browicz var. } \\
\text { orientalis }\end{array}$ & Rosaceae & $\begin{array}{l}\text { Sevtirşk, Yabani elma, } \\
\text { Domuz elması, Ekşi elma }\end{array}$ & Fruit & Dec., Int. & {$[11]$} \\
\hline
\end{tabular}


Erbay et al.

Plants used as painkiller in traditional treatment in Turkey - II Headache

\begin{tabular}{|c|c|c|c|c|c|}
\hline Malva neglecta Wallr. & Malvaceae & $\begin{array}{l}\text { Tolk, Tolga küvi, Tolga } \\
\text { badinga, } \\
\text { Ebegümeci, Ebem } \\
\text { ekmeği, Gömeç }\end{array}$ & Leaf & Inf., Int. & [13] \\
\hline M. sylvestris L. & Malvaceae & $\begin{array}{l}\text { Ebegümeci, Kabaot, } \\
\text { Usluebegüm eci, Develik, } \\
\text { Kedigözü, Evelik, Gömeç }\end{array}$ & Flower & Inf., Int. & [29] \\
\hline $\begin{array}{l}\text { Matricaria chamomilla } \\
\text { L. }\end{array}$ & Asteraceae & $\begin{array}{l}\text { Bopatça, Bupatçe, } \\
\text { Papatça, Papatya, } \\
\text { Keloğlan çiçeği }\end{array}$ & Capitulum & Inf., Int. & {$[15]$} \\
\hline $\begin{array}{l}\text { Melissa officinalis L. } \\
\text { subsp. inodora (Bornm.) } \\
\text { Bornm }\end{array}$ & Lamiaceae & Turunçcuk & Aerial part & Inf. & {$[42]$} \\
\hline $\begin{array}{l}\text { Mentha spicata L. subsp. } \\
\text { tomentosa (Briq.) Harley }\end{array}$ & Lamiaceae & Yarp1z & Leaf & Inf., Int. & [33] \\
\hline $\begin{array}{l}\text { Nasturtium officinale } \\
\text { R.Br. }\end{array}$ & Brassicaceae & $\begin{array}{l}\text { Su gedimesi, Dere } \\
\text { gedimesi, Gadime, Kaz } \\
\text { aya, Tujik, Su teresi, Deli } \\
\text { kereviz, Su kazayağı }\end{array}$ & Whole plant & Eaten, Int. & {$[43]$} \\
\hline $\begin{array}{l}\text { Ononis spinosa L. subsp. } \\
\text { leiosperma (Boiss.) Sirj. }\end{array}$ & Fabaceae & Siyek & Aerial part & $\begin{array}{l}\text { Ashes of burned, } \\
\text { (+egg), Wrapped in a } \\
\text { cloth, Ext. }\end{array}$ & {$[13]$} \\
\hline Origanum onites L. & Lamiaceae & $\begin{array}{l}\text { Kekik, Eşek kekiği, Beyaz } \\
\text { kekik, Deli kekik, Kara } \\
\text { kekik, Taş kekiği, Akbaş } \\
\text { kekik }\end{array}$ & Aerial part & Essential oil, Ext. & {$[22]$} \\
\hline $\begin{array}{l}\text { O. vulgare L. subsp. } \\
\text { hirtum (Link) Ietswaart }\end{array}$ & Lamiaceae & $\begin{array}{l}\text { Kekik, Kekikotu, } \\
\text { Köfteotu, deli kekik, } \\
\text { Karakekik, Güve kekiği, } \\
\text { Yer kekiği, Keklik, Ak } \\
\text { kekik, Ayaklı kekik, } \\
\text { Mercan köşk }\end{array}$ & Leaf & Chewed & [8] \\
\hline Peganum harmala L. & Zygophyllaceae & $\begin{array}{l}\text { Üzerlik, Harmal, Boğir, } \\
\text { Bohol, Mekeç, Nazarlık } \\
\text { otu }\end{array}$ & Seed & Dec./Inf., Int. & [44] \\
\hline $\begin{array}{l}\text { Pistacia terebinthus } \\
\text { L. subsp. palaestina } \\
\text { (Boiss.)Engler }\end{array}$ & Anacardiaceae & $\begin{array}{l}\text { Çöğüre, Menengiç, } \\
\text { Çıtırmak, Sakızlık }\end{array}$ & Leaf & Ext. & {$[14]$} \\
\hline Potentilla anserina $\mathrm{L}$. & Rosaceae & Aslan kecesi & Whole plant & Dec. & {$[45]$} \\
\hline $\begin{array}{l}\text { Primula vulgaris } \\
\text { Huds. subsp. sibthorpii } \\
\text { (Hoffmanss.) W.W.SM. } \\
\text { et. Forest }\end{array}$ & Primulaceae & $\begin{array}{l}\text { Yabani menekşe, Yaban } \\
\text { marulu }\end{array}$ & Leaf and Flower & Inf., Int. & {$[46]$} \\
\hline Prunus $x$ domestica L. & Rosaceae & Huluk & Fruit & Dec., Int. & {$[11]$} \\
\hline Punica granatum L. & Punicaceae & $\begin{array}{l}\text { Hınar, Nar, İnar, Hicaz } \\
\text { narı }\end{array}$ & Fruit & Eaten & {$[47]$} \\
\hline Raphanus sativus L. & Brassicaceae & $\begin{array}{l}\text { Turp, Yabani turp otu, } \\
\text { Deli turp otu, Karaturp }\end{array}$ & Aerial part & Eaten & [9] \\
\hline Rheum ribes $\mathrm{L}$. & Polygonaceae & Revas, Işkın & Root & $\begin{array}{l}\text { Crushed, +Lawsonia } \\
\text { inermis, Ext. }\end{array}$ & {$[11]$} \\
\hline $\begin{array}{l}\text { Rhododendron ponticum } \\
\text { L. subsp. ponticum }\end{array}$ & Ericaceae & $\begin{array}{l}\text { Mor çiçekli orman gülü, } \\
\text { Komar, Ağu }\end{array}$ & $\begin{array}{l}\text { Leaf } \\
\text { Aerial part }\end{array}$ & $\begin{array}{l}\text { Eaten } \\
\text { Ext. }\end{array}$ & $\begin{array}{l}{[39]} \\
{[48 ; 49]}\end{array}$ \\
\hline Rhus coriaria $\mathrm{L}$. & Anacardiaceae & $\begin{array}{l}\text { Sumak, Somak, Somak } \\
\text { otu }\end{array}$ & Leaf & Dec., Ext. & {$[14]$} \\
\hline
\end{tabular}


Erbay et al.

\begin{tabular}{|c|c|c|c|c|c|}
\hline Robinia pseudoacacia L. & Fabaceae & Akasya & Flower & Eaten & {$[50]$} \\
\hline Rosa canina $\mathrm{L}$. & Rosaceae & $\begin{array}{l}\text { Kuşburnu, İt burnu, } \\
\text { Öküzgözü, Yaban gülü, } \\
\text { Kozalak }\end{array}$ & Leaf & Inf., Int. & {$[7 ; 9 ; 29 ; 51]$} \\
\hline Rubia tinctorum L. & Rubiaceae & $\begin{array}{l}\text { Rünas, Boya otu, } \\
\text { Böğürtlen, Kızılboya }\end{array}$ & Root & $\begin{array}{l}\text { Crushed, +Lawsonia } \\
\text { inermis, Ext. }\end{array}$ & {$[11]$} \\
\hline Rubus sanctus Schreber & Rosaceae & $\begin{array}{l}\text { Böğürtlen, Börtlen, } \\
\text { Orman üzümü, Orman } \\
\text { gülü, Kür }\end{array}$ & Fruit & Eaten & {$[15]$} \\
\hline Rumex acetosella $\mathrm{L}$. & Polygonaceae & $\begin{array}{l}\text { Küçük kuzu kulağı, } \\
\text { Ekşi kulak, İlibada, } \\
\text { Kemekulağı }\end{array}$ & Aerial part & Granulated, Int. & {$[52]$} \\
\hline Salix alba $\mathrm{L}$. & Salicaceae & Söğüt & Leaf & Dec./Inf., Int. & {$[8 ; 53]$} \\
\hline S. babylonica L. & Salicaceae & Salkım söğüt & Leaf & Inf., Int. & {$[53]$} \\
\hline Salvia fruticosa Miller & Lamiaceae & $\begin{array}{l}\text { Adaçayı, Almageyik, } \\
\text { Şapla, Yakı otu }\end{array}$ & Aerial part & Inf., Int. & {$[15]$} \\
\hline S. tomentosa Miller & Lamiaceae & $\begin{array}{l}\text { Adaçayı, Şaplağa, Yakı } \\
\text { otu, Şalpa, Sancı otu, Ellik } \\
\text { otu, Kancık, Hoşaflama, } \\
\text { Moşafla, Muşapla }\end{array}$ & Aerial part & Inf., Int. & {$[15]$} \\
\hline Satureja hortensis L. & Lamiaceae & Dağ kekiği, Zahter & Aerial part & Inf., Int. & {$[12]$} \\
\hline $\begin{array}{l}\text { Scutellaria orientalis L. } \\
\text { subsp. pichleri (Stapf) } \\
\text { Edmondson }\end{array}$ & Lamiaceae & Kesel Mahmut & Aerial part & Eaten & {$[11]$} \\
\hline $\begin{array}{l}\text { S. orientalis L. subsp. } \\
\text { virens (Boiss.\& Kotschy) } \\
\text { Edmondson }\end{array}$ & Lamiaceae & Kesel Mahmut, Ververk & $\begin{array}{l}\text { Aerial part } \\
\text { Leaf }\end{array}$ & $\begin{array}{l}\text { Eaten } \\
\text { Dec., Int. }\end{array}$ & $\begin{array}{l}{[11]} \\
{[54]}\end{array}$ \\
\hline Sinapis arvensis $\mathrm{L}$. & Brassicaceae & $\begin{array}{l}\text { Hardal, Sarı hardal, } \\
\text { Hardal otu, Gıcı, Kara } \\
\text { hardal }\end{array}$ & $\begin{array}{l}\text { Root } \\
\text { Leaf }\end{array}$ & $\begin{array}{l}\text { Chewed, Int. } \\
\text { Not stated }\end{array}$ & $\begin{array}{l}{[13]} \\
{[55]}\end{array}$ \\
\hline Solanum tuberosum L. & Solanaceae & $\begin{array}{l}\text { Patates } \\
\text { Gumpir }\end{array}$ & $\begin{array}{l}\text { Tuber } \\
\text { Tuber cortex }\end{array}$ & $\begin{array}{l}\text { Sliced, Ext. } \\
\text { Ext. }\end{array}$ & $\begin{array}{l}{[9 ; 40 ; 56 ; 57 ; 58]} \\
{[14]}\end{array}$ \\
\hline $\begin{array}{l}\text { Stachys lavandulifolia } \\
\text { Vahl. }\end{array}$ & Lamiaceae & Devegülü, Tüylü çay & Flower & Inf., Int. & [59] \\
\hline $\begin{array}{l}\text { S. mardinensis (Post) } \\
\text { R.Mill. }\end{array}$ & Lamiaceae & Kaya pungu, Punge tehta & Whole plant & Not stated & {$[60]$} \\
\hline $\begin{array}{l}\text { Stellaria media (L.) Vill. } \\
\text { subsp. media }\end{array}$ & Caprifoliaceae & Zurzula & Stem and leaf & Cooked, Eaten & {$[24]$} \\
\hline Tanacetum balsamita $\mathrm{L}$. & Asteraceae & Marsuvan otu & Aerial part & Inf., Int. & {$[30]$} \\
\hline $\begin{array}{l}\text { Thymbra spicata L. var. } \\
\text { spicata }\end{array}$ & Lamiaceae & $\begin{array}{l}\text { Seyil kekiği, Kara kekik, } \\
\text { Karaçekme, Zahter, Eşek } \\
\text { zahteri, Kırçayı, Bayır } \\
\text { kekiği, Karabaş otu, Kaya } \\
\text { kekiği, Şeker otu }\end{array}$ & $\begin{array}{l}\text { Aerial part } \\
\text { Flower }\end{array}$ & $\begin{array}{l}\text { Inf., Int. } \\
\text { Dec. }\end{array}$ & $\begin{array}{l}{[9 ; 27]} \\
{[9]}\end{array}$ \\
\hline $\begin{array}{l}\text { Tilia argentea Desf. ex } \\
\text { DC. }\end{array}$ & Tiliaceae & Ihlamur & Flower & Dec., Int. & {$[29]$} \\
\hline Urtica pilulifera L. & Urticaceae & Isırgan, Istırgaç & Aerial part & Inf., Int./Ext. & [9] \\
\hline Viola occulta Lehm. & Violaceae & Menekșe & Flowering leaf & Dec., Int. & {$[50]$} \\
\hline $\begin{array}{l}\text { Viscum album L. subsp. } \\
\text { album }\end{array}$ & Loranthaceae & $\begin{array}{l}\text { Ökse otu, Büvelek otu, } \\
\text { Burç, Buruç, Güvelek, } \\
\text { Puruş }\end{array}$ & $\begin{array}{l}\text { Aerial part } \\
\text { Fruit }\end{array}$ & $\begin{array}{l}\text { Dec., Mouthwash } \\
\text { Dec., Int. }\end{array}$ & $\begin{array}{l}{[9]} \\
{[61]}\end{array}$ \\
\hline Vitex agnus-castus L. & Verbenaceae & Hayıt, Ayıt & Leaf & Ext. & {$[41]$} \\
\hline
\end{tabular}

Inf: Infusion, Dec: Decoction, Int: Internal, Ext: External 
Turkey has many plants used by public for the treatment of various diseases. The usage of these folk medicine which is prepared by these plants has been transferred from generation to generation. Ethnobotanical studies carried out by traditional methods of treatment are recorded and this information is aimed to contribute to the development of the drug.

This study reached 88 taxa traditionally used against headache in Turkey. These plants are mainly from Lamiaceae (17 taxa), Rosaceae (10 taxa), Asteraceae (9 taxa), Araceae (4 taxa), Cucurbitaceae ( 3 taxa) and Malvaceae (3 taxa) families (Figure 1).

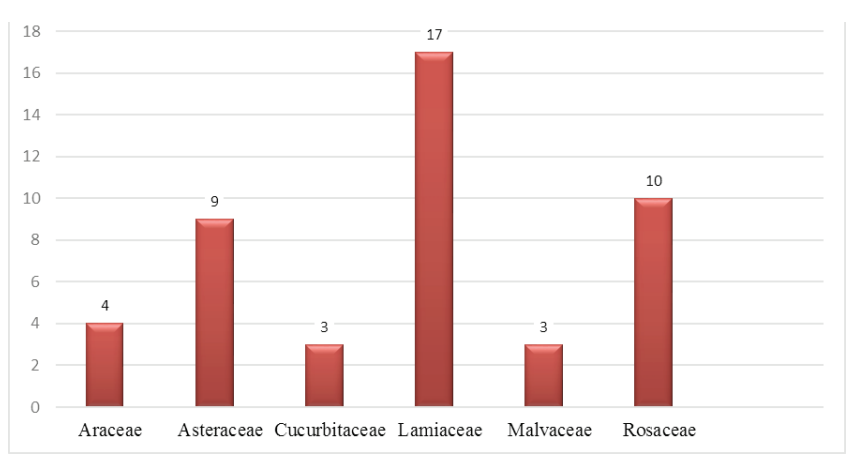

Figure 1. Graph of main families used in traditional treatment against headache in Turkey.

Most used plants as painkiller in traditional treatment against headache in Turkey are Solanum tuberosum, Rosa canina, Allium sativum, Bellis perennis, Cydonia oblonga, Ecballium elaterium, Juglans regia, Lavandula stoechas, Thymbra spicata var. spicata. Some of the plants in the table (Arum sp., Buxus sempervirens, Ecballium elaterium, Fritillaria acmopetala, Glaucium leiocarpum, Hypericum scabrum, Inula heterolepsis, Laurocerasus officinalis, Peganum harmala, Primula vulgaris subsp. sibthorpii, Prunus domestica, Rhododendron ponticum subsp. ponticum, Robinia pseudoacacia, Tanacetum balsamita, Viscum album) are known as poisonous. It is important to note that some of the poisonous plants are being used internally.

The most used families such as Lamiaceae, Rosaceae and Asteraceae are rich from essential oil. Essential oils have sedative and antispazmodic effects. Because of these effects, the plants belong these families use against headache. These compounds (especially menthol) provide constriction of loose veins. In the brain, the veins that relax and compress the nerves become narrowed by the effect of these compounds and the headache is reduced. Except for this effect, plants used are also known to contain analgesic, antiinflammatory and tranquilizing compounds. These effects can also treat simple causes underlying the headache. The most commonly used parts of plants are; leaves, aerial parts, fruits, flowers and roots. Generally, parts such as leaves, flowers and aerial parts are used externally or by wrapping around after decoction or infusion is made. Parts such as root, seed and aerial parts are crushed and put into a mush and then wrapped around head and used externally. Fruits fresh or dried are usually eaten against headache.

We hope that this study will contribute to the development of new drugs to be used in the treatment of headache.

\section{REFERENCES}

[1] Yüceer S. MSc Thesis. Knowledge of nursing students related fifth vital sign pain and postoperative pain. Nursing Department, Faculty of Health Sciences, Hacettepe University, Ankara, 2008.

[2] Erdine S. İstanbul Ağrı Merkezi. Available in: www.agritr. com./html/algoloji.html [Accessed: 12.05.2015].

[3] Güner A, Özhatay N, Ekim T, Başer KHC, Flora of Turkey and the East Islands, vol. 11, Edinburgh University Press, Edinburgh, 2000.

[4] Özhatay N, Kültür Ş, Gürdal B. Check-list of additional taxa to the supplement flora of Turkey VI. J Fac Pharm Istanbul. 2013; 43 (1): 33-82.

[5] Özhatay N, Kültür Ş, Gürdal B. Check-list of additional taxa to the supplement flora of Turkey VII. J Fac Pharm Istanbul. 2015; 45 (1): 61-86.

[6] Kalafatçılar ÖA, Kalafatçılar Aİ, Bitkiler ve Sağlık - Fitoterapi-, Sidas Medya Ltd. Şti., İzmir, 2010.

[7] Sezik E, Yeşilada E, Honda G, Takaishi Y, Takeda Y, Tanaka T. Traditional medicine in Turkey X. Folk medicine in Central Anatolia. J Ethnopharmacol. 2001; 75 (2-3): 95-115. https:// doi.org/10.1016/S0378-8741(00)00399-8

[8] Kültür Ş. Medicinal plants used in Kırklareli province (Turkey). J Ethnopharmacol. 2007; 111: 341-364. https://doi. org/10.1016/j.jep.2006.11.035

[9] Sargin SA. PhD Thesis. Agricultural biodiversity and ethnobotanical survey of Alaşehir (Manisa) and its surrounding area. Department of Biology, Balıkesir University, Balıkesir, 2013.

[10] Şahin Yiğit S. MSc Thesis. Medicinal plants sold in Gaziantep herbalists and their ethnobotanical aspects. Department of Biology, Gaziantep University, Gaziantep, 2014.

[11] Mükemre M. MSc Thesis. Ethnobotanical features of Konalga, Sırmalı, Dokuzdam villages (Çatak/ Van) and their vicinity. Department of Biology, Yüzüncü Yll University, Van, 2013.

[12] Furkan MK. MSc Thesis. The ethnobotanical properties of some plants growing in Adiyaman. Department of Biology, Adıyaman University, Adıyaman, 2016.

[13] Şenkardeş İ. PhD Thesis. Ethnobotanical investigations in southern districts (Acıgöl, Derinkuyu, Gülşehir, NevşehirCentral district, Ürgüp) of Nevşehir. Department of Pharmaceutical Botany, Faculty of Pharmacy, Marmara University, İstanbul, 2014. 
[14] Güneş S. MSc Thesis. Karaisalı (Adana), and villages natural plants used by the public, ethnobotanical respect investigation. Department of Biology, Niğde University, Niğde, 2010.

[15] Özdemir Nath E. PhD Thesis. An ethnobotanical study in Savaştepe and Kepsut region (Balıkesir). Department of Pharmaceutical Botany, Institue of Health Science, Istanbul University, İstanbul, 2016.

[16] Kaval İ. MSc Thesis. Ethnobotanical features of Geçitli (Hakkari) and surroundings. Department of Biology, Yüzüncü Yll University, Van, 2011.

[17] Tuzlacı E, Eryaşar Aymaz P. Turkish folk medicinal plants, Part IV: Gönen (Balıkesir). Fitoterapia 2001; 72: 323-343. https://doi.org/10.1016/S0367-326X(00)00277-X

[18] Keklik Koçoğlu T, Çubukçu B, Özhatay N. Folkloric drugs of Konya and Karaman. J Tradit Folkloric Drugs 1996; 3(1): 1-71.

[19] Uzun E, Sarıyar G, Adsersen A, Karakoç B, Ötük G, Oktayoğlu E, Prrldar S. Traditional medicine in Sakarya province (Turkey) and antimicrobial activities of selected species, J Ethnopharmacol 2004; 95: 287-296. https://doi.org/10.1016/j. jep.2004.07.013

[20] Ayan Ö. MSc Thesis. Antimicrobial activities of some edible plant taxa which are used as ethnobotonical purpose in Kastamonu region, on some food pathogens. Department of Forest Engineering, Kastamonu University, Kastamonu, 2015.

[21] Everest A, Öztürk E. Focusing on the ethnobotanical uses of plants in Mersin and Adana provinces (Turkey). J Ethnobiol Ethnomed 2005; 1: 6. doi: 10.1186/1746-4269-1-6

[22] Gürdal B. MSc Thesis. Ethnobotanical study in Marmaris district (Muğla). Department of Pharmaceutical Botany, Istanbul University, İstanbul, 2010.

[23] Toksoy D, Bayramoglu M, Hacisalihoglu S. Usage and the economic potential of the medicinal plants in Eastern Black Sea Region of Turkey. J Environ Biol 2010; 31(5): 623-628.

[24] Sağıroğlu M, Arslantürk A, Akdemir ZK, Turna M. An ethnobotanical from Hayrat (Trabzon) and Kalkandere (Rize/ Turkey). Biol Divers Conserv 2012; 5 (1): 31-43.

[25] Akgül A. MSc Thesis. Ethnobotany at Midyat (Mardin). Department of Biology, Ege University, İzmir, 2008.

[26] Ezer N, Avcı K. Çerkeş (Çankırı) Yöresinde kullanılan halk ilaçları. Hacettepe Univ J Fac Pharm 2004; 24(2): 67-80.

[27] Emre Bulut G. PhD Thesis. Ethnobotanical investigations in Bayramiç (Çanakkale). Department of Pharmaceutical Botany, Marmara University, İstanbul, 2008.

[28] Ecevit Genç G. MSc Thesis. Çatalca yöresinde etnobotanik bir araştırma. Department of Pharmaceutical Botany, Istanbul University, Istanbul, 2003.

[29] Akalın E. The plants of Tekirdağ region that are used as medicinal purposes and food. J Tradit Folkloric Drugs 1998; 5(1)7: 1-96.

[30] Altundağ E, Öztürk M. Ethnomedicinal studies on the plant resources of east Anatolia Turkey. Procedia Soc Behav Sci 2011; 19: 756-777. https://doi.org/10.1016/j.sbspro.2011.05.195
[31] Alpaslan Z. MSc Thesis. The ethnobotanical properties of Ergan mountain (Erzincan). Department of Biology, Erzincan University, Erzincan, 2012.

[32] Korkmaz M, Alpaslan Z. Ergan dağı (Erzincan - Türkiye)'nın etnobotanik özellikleri. Bağbahçe Bilim Derg 2014; 1(3): 1-31.

[33] Erdoğan R. MSc Thesis. Ethnobotanical features some of the wild on the Sarıveliler (Karaman) and its environment ethnobotanic. Department of Biology, Selçuk University, Konya, 2011.

[34] Tuzlacı E, Doğan A. Turkish folk medicinal plants, IX: Ovacık (Tunceli). Marmara Pharm J 2010; 14: 136-143.

[35] Tetik F. MSc Thesis. A research on the ethnobotanical valued plants in Malatya province. Firat University, Elazığ, 2011.

[36] Yeşil Y. MSc Thesis. Ethnobotanical study in Kürecik district (Malatya/ Akçadağ). Department of Pharmaceutical Botany, İstanbul University, İstanbul, 2007.

[37] Aktan T. MSc Thesis. The Ethnobotanical examine in the villages of Yenişehir (Bursa). Department of Biology, Celal Bayar University, Manisa, 2011.

[38] Doğru Koca A, Yıldırımlı Ş. Ethnobotanical properties of Akçakoca district in Düzce (Turkey). Hacettepe J Biol Chem 2010; 38(1): $63-69$.

[39] Polat R, Cakilcioglu U, Kaltalioglu K, Denizhan M, Ulusan MD. An ethnobotanical study on medicinal plants in Espiye and its surrounding (Giresun - Turkey). J Ethnopharmacol 2015; 163: 1-11. doi: 10.1016/j.jep.2015.01.008.

[40] Metin A. Msc Thesis. Ethnobotanical features of plants in Mut (Mersin) and its environments. Department of Biology, Selçuk University, Konya, 2009.

[41] Polat R. PhD Thesis. Agricultural biodiversity and ethnobotanical research in the Havran and Burhaniye regions of Balıkesir. Department of Biology, Balıkesir University, Balıkesir, 2010.

[42] Demirci S. MSc Thesis. Ethnobotanical study in Andırın (Kahramanmaraş) district. Department of Pharmaceutical Botany, İstanbul University, İstanbul, 2010.

[43] Kılıç Ö, Bağcı E. An ethnobotanical survey of some medicinal plants in Keban (Elazığ - Turkey). J Med Plants 2013; 7(23): 1675-1684. DOI: 10.5897/JMPR2013.4451

[44] Oral ÇD. Msc Thesis. Ethnobotanical studies on folk medicines used in Konya. Program Phytotherapy, Gazi University, Ankara, 2007.

[45] Güneş F, Özhatay N. An ethnobotanical study from Kars (Eastern) Turkey. Biol Divers Conserv 2011; 4(1): 30-41.

[46] Poyraz Kayabaşı N. MSc Thesis. The ethnobotanical examine in the villages of Manyas and Manyas. Department of Biology, Balıkesir University, Balıkesir, 2011.

[47] Gençay A. MSc Thesis. Ethnobotanical aspects of Cizre (Şırnak). Department of Biology, Yüzüncü Yll University, Van, 2007.

[48] Saraç DU. Ethnobotanic features of Rize province. MSc Thesis. Forest Engineering Graduate Program, Karadeniz Technical University, Trabzon, 2013.

[49] Saraç DU, Özkan ZC, Akbulut S. Ethnobotanic features of Rize/Turkey province. Biol Divers Conserv 2013; 6(3): 57-66. 
[50] Koyuncu $\mathrm{O}, \mathrm{PhD}$ Thesis. Investigations of floristical and etnobotanical aspect of Geyve (Sakarya) and its environs. Department of Biology, Eskişehir Orhangazi University, Eskişehir, 2005.

[51] Tuzlacı E, Tolon E. Turkish folk medicinal plants. Part III: Şile (Istanbul). Fitoterapia 2000; 71: 673-685.

[52] Yeşilada E, Honda G, Sezik E, Tabata M, Fujita T, Tanaka T, Takeda Y, Takaishi Y. Traditional medicine in Turkey. V. Folk medicine in inner Taurus Mountains. J Ethnopharmacol 1995; 46: 133-152.

[53] Bulut G. Folk medicinal plants of Silivri (İstanbul, Turkey). Marmara Pharm J 2011; 15: 25-29. DOI : 10.12991/201115441

[54] Balos MM. MSc Thesis. The flora and ethnobotany of the region between Zeytinbahçe and Akarçay (Birecik). Department of Biology, Harran University, Şanlıurfa, 2007.

[55] Yapıcı İ, Hoşgören H, Saya Ö. Kurtalan (Siirt) ilçesinin etnobotanik özellikleri. Dicle Univ J Ziya Gökalp Fac Educ 2009; 12: 191-196.
[56] Keskin L. MSc Thesis. Kadınhanı (Konya) ve çevresinde yetişen bitkilerin etnobotanik özellikleri. Department of Biology, Konya Selçuk University, Konya, 2011.

[57] Tekin S. MSc Thesis. Ethnobotanic Aspects of Üzümlü (Erzincan) Town. Department of Biology, Erzincan University, Erzincan, 2011.

[58] Ayandın H. MSc Thesis. The Ethnobotanic Characteristics of the region among Avşar, Şabanözü and Çile Mountain (Polatlı / Ankara). Department of Biology, Selçuk University, Konya, 2010.

[59] Özdemir E, Alpınar K. An ethnobotanical survey of medicinal plants in western part of central Taurus Mountains: Aladaglar (Niğde - Turkey). J Ethnopharmacol 2015; 166:53-65.

[60] Korkut MM. MSc Thesis. The flora and ethnobotany of Arat mountain (Şanlıurfa). Department of Biology, Harran University, Şanlıurfa, 2006.

[61] Kahraman A, Tatlı A. Umurbaba Dağı (Eşme - Uşak) ve çevresindeki bazı bitkilerin mahalli adları ve etnobotanik özellikleri. OT Sistematik Bot Derg 2004; 11(2): 147-154. 